\title{
Localização inicial da ponta de cateter central de inserção periférica (PICC) em recém-nascidos*
}

\author{
INITIAL PLACEMENT OF THE PERIPHERALLY INSERTED CENTRAL CATHETER'S \\ TIP IN NEONATES
}

\author{
LOCALIZACIÓN INICIAL DE LA PUNTA DEL CATÉTER CENTRAL DE INSERCIÓN \\ PERIFÉRICA (PICC) EN RECIÉN NACIDOS
}

\author{
Patrícia Ponce de Camargo ${ }^{1}$, Amélia Fumiko Kimura², Edi Toma ${ }^{3}$, Maria Alice Tsunechiro ${ }^{4}$
}

\begin{abstract}
RESUMO
Estudo transversal com coleta prospectiva de dados, que objetivou identificar o posicionamento inicial da ponta do cateter central de inserção periférica (PICC) e verificar a prevalência de sucesso de sua inserção em neonatos. Os dados foram coletados no berçário anexo à maternidade do Hospital das Clínicas da Faculdade de Medicina da Universidade de São Paulo, entre março e setembro de 2006. Dos 37 neonatos submetidos à inserção do cateter PICC, a taxa de sucesso no procedimento foi de $72,3 \%$ (27 neonatos); destes, quatro $(14,8 \%)$ estavam com as pontas dos cateteres alojadas nas veias axilar ou inominada; outros três $(11,1 \%)$, alojadas em veia jugular. Estes cateteres foram removidos por desvio de trajeto. $13(48,2 \%)$ estavam com as pontas alojadas em átrio direito, cujos cateteres foram tracionados para reposicionamento da ponta para a veia cava superior.
\end{abstract}

\section{DESCRITORES}

Cateterismo periférico.

Veias cavas.

Recém-nascido.

Prematuro.

\begin{abstract}
This is a cross-sectional study aiming to identify the initial tip position of peripherally inserted central catheters (PICC) and to verify the prevalence of success in inserting such catheters in neonates. The study was carried out in the neonatal care unit of Hospital das Clínicas, Universidade de São Paulo. Data were collected prospectively from March to September 2006. 37 neonates underwent PICC insertion were included in the study. The rate of success for this procedure was $72.3 \%$ ( 27 neonates). Of them, four (14.8\%) had the catheter tips placed in the axilary or inominate veins. Three others (11.1\%) had them placed in a jugular vein. When these catheters were removed, $13(48.2 \%)$ catheter tip were placed in the right atrium, and they were relocated to the superior vena cava.
\end{abstract}

\section{KEY WORDS}

Catheterization, peripheral.

Venae cavae.

Infant, newborn.

Infant, premature.

\section{RESUMEN}

Estudio transversal con recolección prospectiva de datos. La finalidad fue identificar la posición inicial de la punta del catéter central de inserción periférica (PICC) y verificar la prevalencia de éxitos durante su introducción en neonatos. Los datos fueron recolectados en un servicio de neonatología anexo a la maternidad del Hospital de las Clínicas de la Facultad de Medicina de la Universidad de São Paulo, entre marzo y setiembre del 2006. De los 37 neonatos sometidos a introducción del catéter PICC, la tasa de éxito fue de $72.3 \%$ ( 27 neonatos), de ellos, cuatro (14.8\%) estaban con las puntas de los catéteres alojadas en las venas axilar o no determinada, tres (11.1\%) localizadas en la vena yugular. Siendo estos últimos retirados por desviación en su trayecto. El $48.2 \%$ (13) se encontraba con las puntas en el atrio derecho, siendo estos catéteres nuevamente posicionados en la vena cava superior.

\section{DESCRIPTORES \\ Cateterismo periférico. \\ Venas cavas. \\ Recién nacido. \\ Prematuro.}

* Extraído da dissertação "Procedimento de inserção, manutenção e remoção do cateter central de inserção periférica em neonatos", Escola de Enfermagem, Universidade de São Paulo, 2007. ${ }^{1}$ Mestranda do Programa de Pós-Graduação da Escola de Enfermagem, Universidade de São Paulo (EEUSP). Enfermeira do Berçário Anexo à Maternidade do Hospital das Clínicas da Faculdade de Medicina, Universidade de São Paulo. São Paulo, SP, Brasil. patrícia.ponce@uol.com.br

${ }^{2}$ Enfermeira. Professora Doutora do Departamento de Enfermagem Materno-Infantil e Psiquiátrica da Escola de Enfermagem, Universidade de São Paulo (EEUSP). São Paulo, SP, Brasil. fumiko@usp.br ${ }^{3}$ Enfermeira. Doutora em Enfermagem. Chefe de Enfermagem do Berçário Anexo à Maternidade do Hospital das Clínicas da Faculdade de Medicina, Universidade de São Paulo. São Paulo, SP, Brasil. editoma@uol.com.br ${ }^{4}$ Enfermeira. Professora Doutora do Departamento de Enfermagem Materno-Infantil e Psiquiátrica da Escola de Enfermagem, Universidade de São Paulo (EEUSP). São Paulo, SP, Brasil. tamnami@usp.br 


\section{INTRODUÇÃO}

O cateter central de inserção periférica (PICC) cada vez mais tem sido utilizado no cuidado de pacientes em estado crítico nas unidades de terapia intensiva neonatal (UTIN), particularmente, os recém-nascidos pré-termos de muito baixo peso ${ }^{(1-3)}$.

O PICC permite manter o acesso venoso por período prolongado e infundir medicamentos, soluções hipertônicas e nutrição parenteral total (NPT) em veias centrais de forma segura(4-5).

Nas unidades de cuidado intensivo é onde mais se utilizam os cateteres PICC, sendo os enfermeiros os profissionais responsáveis pela sua inserção. Assim, cada vez mais os enfermeiros buscam capacitar-se para o exercício desta prática.

No Brasil, a atribuição da competência técnica e legal do enfermeiro para exercer a prática de manipulação do cateter PICC foi definida na Resolução n² 258/2001 do Conselho Federal de Enfermagem.

O crescimento e a expansão da função da enfermagem são necessários e desejáveis ${ }^{(6)}$. No entanto, a incorporação de novos procedimentos na prática profissional deve ser monitorada a fim de identificar as possíveis complicações decorrentes destas práticas.

Não obstante, os inúmeros benefícios atribuídos ao uso do cateter PICC, os profissionais devem estar atentos aos riscos envolvidos no uso deste dispositivo, que está associado a algumas complicações que podem ocorrer na inserção, enquanto o cateter percorre o trajeto venoso, durante a manutenção e sua remoção. Tais complicações ocorrem por problemas mecânicos, como: obstrução, ruptura do cateter, perfuração de vaso, extravasamento, trombose, hidrotórax, entre outros, e problemas infecciosos, sobretudo, a sepse sistêmica relacionada ao cateter $\operatorname{PICC}^{(1,5)}$.

O sucesso na inserção do PICC é obtido quando a ponta do cateter posiciona-se centralmente, isto é, em veia cava superior. Se a ponta progredir para além da veia cava superior, manobras de tração serão aplicadas no cateter para seu reposicionamento ${ }^{(7)}$.

Pontas de cateteres posicionadas centralmente estão associadas com baixas taxas de complicações comparadas aos cateteres não centrais ${ }^{(2)}$. Assim, a manutenção da ponta do cateter em posição central é de suma importância para reduzir o risco de complicações decorrentes do uso desse dispositivo.

O cateter PICC tem sido amplamente utilizado para administração de nutrição parenteral e antibiótico; entretanto, quando a ponta não está posicionada centralmente al- gumas complicações podem ocorrer, tais como: tromboflebite, flebites e oclusões ${ }^{(8)}$.

A migração da ponta do cateter PICC é um problema comum e conhecido entre os neonatologistas, podendo levar a um quadro letal de efusão pericárdica, tamponamento secundário à perfuração miocárdica. Pontas de cateteres posicionadas em átrio direito ou sua migração para dentro do átrio direito são apontadas como prováveis causas destas complicações ${ }^{(9)}$.

Certificar-se da localização inicial da ponta do cateter PICC, após o procedimento de sua inserção, é uma medida de segurança recomendada aos profissionais que atuam no manejo do cateter PICC.

A taxa de sucesso no correto posicionamento inicial da ponta do cateter PICC é a chave para determinar a necessidade de outras manobras na instalação do cateter. Quanto menor a taxa de sucesso no correto posicionamento inicial da ponta do cateter, maior será a freqüência de sua manipulação.

Assim sendo, cada serviço necessita monitorar a própria taxa de sucesso no posicionamento da ponta do cateter PICC, a fim de detectar os fatores causais e intervenientes das falhas e implementar medidas para melhorar a taxa de sucesso desse procedimento.

\section{OBJETIVOS}

- Identificar o posicionamento inicial da ponta do cateter central de inserção periférica;

- Verificar a prevalência de sucesso na inserção do cateter central de inserção periférica.

\section{MÉTODO}

\section{Desenho do estudo}

Estudo transversal com coleta prospectiva dos dados sobre a prática de enfermeiras no procedimento de inserção do cateter central de inserção periférica em recém-nascidos internados em uma Unidade de Terapia Intensiva Neonatal.

\section{Local do estudo}

A pesquisa foi desenvolvida no Berçário Anexo à Maternidade do Hospital das Clínicas da Faculdade de Medicina da Universidade de São Paulo que conta com um quadro de 17 enfermeiras, sete delas com certificação no procedimento de instalação de cateter PICC conferida pela Sociedade Brasileira de Terapia Intensiva (SOBETI). A decisão de inserir o cateter PICC é tomada pela equipe que assiste o recém-nascido. Antes da realização do procedimento de 
inserção do cateter, o médico de plantão e a enfermeira verificam os resultados laboratoriais do recém-nascido, avaliam os riscos e os benefícios de submetê-lo ao procedimento naquele momento. As etapas do procedimento de inserção, manutenção e remoção do cateter PICC seguem as recomendações adotadas pelo curso oferecido pela Sociedade Brasileira de Enfermeiros em Terapia Intensiva.

\section{População}

No estudo, foram incluídos todos procedimentos de implantação do cateter PICC realizados por enfermeiras, ocorridos entre março e setembro de 2006. Os critérios de elegibilidade foram: ter consentimento dos pais dos recémnascidos submetidos ao procedimento de inserção do cateter PICC para utilizar informações do prontuário do recém-nascido e consentimento das enfermeiras que instalaram o cateter PICC em participar do estudo.

\section{Variáveis do estudo}

Analisaram-se as variáveis relacionadas à caracterização da população de recém-nascidos (idade gestacional ao nascimento; peso na data da inserção do PICC; sexo; diagnóstico clínico; terapia parenteral instituída pelo cateter PICC) e as variáveis relacionadas à inserção do cateter (tipo de material, calibre, posicionamento inicial da ponta do cateter, sucesso no procedimento de instalação do PICC).

\section{Instrumento de coleta dos dados}

Os dados coletados foram registrados em formulário impresso contendo duas partes: dados de identificação e características do recém-nascido e dados sobre o procedimento de inserção do cateter PICC.

\section{Procedimento de coleta dos dados}

Os dados foram obtidos pela consulta aos prontuários dos recém-nascidos e observação do procedimento de inserção do cateter realizado pelas enfermeiras. Antes do início do procedimento, solicitou-se consentimento dos pais ou responsável pelo recém-nascido e da enfermeira responsável pela prática da inserção do cateter PICC no recém-nascido. Em observância às determinações da Resolução 196/96, do Conselho Nacional de Saúde, órgão que dispõe sobre as diretrizes e normas regulamentadoras das pesquisas envolvendo seres humanos, no Brasil, o projeto de pesquisa com os respectivos Termos de Consentimento Livre e Esclarecido (responsável pelo recém-nascido e enfermeira) foram submetidos à apreciação e aprovação pelo Comitê de Ética em Pesquisa da Diretoria Clínica do Hospital das Clínicas da Faculdade de Medicina da Universidade de São Paulo (HCFMUSP), cadastrado na Comissão Nacio- nal de Ética em Pesquisa (CONEP). Após a emissão da Carta de Aprovação concedida pelo Comitê de Ética em Pesquisa (Protocolo no 00526200604 de 24/02/2006), a coleta dos dados foi iniciada.

\section{Organização, tratamento e análise dos dados}

Os dados foram armazenados em planilha eletrônica Microsoft Excel e transportados para o programa Epi-Info 3.3.2 para seu processamento. Para as variáveis quantitativas, calcularam-se médias, medianas, valores máximos, mínimos e desvio-padrão. Os dados foram agrupados em freqüência absoluta e relativa e apresentados em forma de Tabelas.

\section{RESULTADOS}

Quanto à caracterização dos recém-nascidos submetidos ao procedimento de inserção do cateter PICC (Tabela 1 e 2) verifica-se que houve predominância de recém-nascidos do sexo masculino, que nasceram com até 32 semanas de idade gestacional (média 31,6 semanas), com peso igual ou inferior a 1.500 gramas (média do peso, 1.289 gramas) e o cateter foi inserido ainda na primeira semana de vida do recém-nascido para infusão de Nutrição Parenteral Total.

Tabela 1 - Caracterização dos recém-nascidos submetidos ao procedimento de inserção do cateter PICC, Berçário Anexo à Maternidade do HCFMUSP - São Paulo - 2006

\begin{tabular}{|c|c|c|}
\hline VARIÁVEIS & $\mathbf{N}=\mathbf{3 7}$ & $\%$ \\
\hline \multicolumn{3}{|l|}{ Sexo } \\
\hline Masculino & 22 & 59,4 \\
\hline Feminino & 15 & 40,6 \\
\hline \multicolumn{3}{|l|}{ Idade Gestacional ao nascimento } \\
\hline$\leq 32$ semanas & 23 & 62,2 \\
\hline$>32$ semanas & 14 & 37,8 \\
\hline \multicolumn{3}{|l|}{ Idade na data do procedimento } \\
\hline Um a três dias & 18 & 48,7 \\
\hline Quatro a sete dias & 14 & 37,8 \\
\hline Oito a onze dias & 3 & 8,1 \\
\hline Doze a quinze dias & 2 & 5,4 \\
\hline \multicolumn{3}{|l|}{ Peso na data a inserção do PICC } \\
\hline$\leq 1500 \mathrm{~g}$ & 27 & 72,3 \\
\hline$>1500 \mathrm{~g}$ & 10 & 27,7 \\
\hline \multicolumn{3}{|l|}{ Diagnóstico } \\
\hline Síndrome do desconforto respiratório & 21 & 56,8 \\
\hline Síndrome do pulmão umido & 11 & 29,7 \\
\hline Cardiopatia congenital & 2 & 5,4 \\
\hline Enterocolite necrosante & 2 & 5,4 \\
\hline Síndrome da aspiração meconial & 1 & 2,7 \\
\hline \multicolumn{3}{|l|}{ Indicação para uso do cateter PICC } \\
\hline Infusão de Nutrição Parenteral Total & 35 & 94,6 \\
\hline Infusão de Prostaglandina E1 & 2 & 5,4 \\
\hline
\end{tabular}


Tabela 2 - Medidas descritivas das variáveis de caracterização dos recém-nascidos submetidos à implantação do PICC, Berçário Anexo à Maternidade do HCFMUSP - São Paulo - 2006

\begin{tabular}{|c|c|c|c|c|c|c|}
\hline Variável & Média & Mediana & Moda & $\begin{array}{c}\text { Valor } \\
\text { Máximo }\end{array}$ & $\begin{array}{l}\text { Valor } \\
\text { Mínimo }\end{array}$ & $\begin{array}{l}\text { Desvio } \\
\text { Padrão }\end{array}$ \\
\hline $\begin{array}{l}\text { Idade gestacional ao } \\
\text { nascimento }\end{array}$ & 31,6 & 31,4 & 27,8 & 39,8 & 25,8 & 3,6 \\
\hline Peso em gramas & $1.289,2$ & $1.200,0$ & $1.040,0$ & $2.570,0$ & 525,0 & 541,5 \\
\hline Idade cronológica em dias & 4,3 & 4,0 & 2,0 & 15,0 & 1,0 & 3,1 \\
\hline
\end{tabular}

Com relação ao tipo de material do cateter PICC, foram utilizados cateteres de poliuretano em 35 (94,6\%) recémnascidos, mais rígido e o de silicone, mais flexível, em apenas dois $(5,4 \%)$ recém-nascidos.

Todos os cateteres tinham calibre 2 French com comprimento que variou de 48 a 50 centímetros.

Dos trinta e sete recém-nascidos submetidos ao procedimento de instalação do cateter PICC, o cateter foi inserido com sucesso em $72,3 \%$ (27 recém-nascidos), outros $20,7 \%$ (dez neonatos) o procedimento de inserção resultou em fracasso relacionado à punção venosa, à não progressão do cateter, entre outros motivos.

Antes de inserir o cateter PICC, mensura-se sua extensão a ser introduzida, considerando o sítio da inserção à veia cava superior. No entanto, no procedimento de inserção do cateter, a ponta pode progredir para um local não planejado, extraviando-se para outra ramificação venosa, ou mesmo, posicionando-se em local anterior ou posterior ao planejado. A Tabela 3 mostra o posicionamento inicial da ponta do cateter PICC identificada por imagem radiográfica.

Tabela 3 - Posicionamento inicial da ponta do cateter PICC, Berçário Anexo à Maternidade do HCFMUSP - São Paulo - 2006

\begin{tabular}{lrc}
\hline $\begin{array}{l}\text { Posicionamento inicial da } \\
\text { ponta do cateter PICC }\end{array}$ & N & \% \\
\hline Não central & 13 & 48,2 \\
$\quad$ Átrio direito & 4 & 14,8 \\
$\quad$ Veia axilar ou veia inominada & 3 & 11,1 \\
$\quad$ Veia jugular & & \\
Central & 7 & 25,9 \\
$\quad$ Veia cava superior & $\mathbf{2 7}$ & $\mathbf{1 0 0 , 0}$ \\
\hline Total &
\end{tabular}

Os dados da Tabela 3 mostram que, em apenas sete $(25,9 \%)$ recém-nascidos, as pontas dos cateteres encontravam-se em veia cava superior. Dos 27 cateteres inseridos, quatro $(14,8 \%)$ estavam com as pontas em região periférica, ou seja, alojadas em veia axilar ou inominada, outros três $(11,1 \%)$ estavam com as pontas alojadas em veia jugular e foram removidos por desvio de trajeto. Chama a atenção que, em 13 (48,2\%) recém-nascidos, as imagens radiológicas mostravam que as pontas dos cateteres estavam alojadas em átrio direito, levando a concluir que a medida da extensão do cateter entre o sitio de inserção à veia cava superior havia sido super dimensionada, uma vez que a ponta ultrapassou a veia cava superior e alojou-se em átrio direito, necessitando de manobras de tracionamento para reposicionar a ponta em local correto. A seguir, na Tabela 4 , são apresentados dados referentes à localização da ponta dos cateteres nos recém-nascidos submetidos à tração.

Tabela 4 - Local alojado pela ponta do cateter PICC após manobra de tração, Berçário Anexo à Maternidade do HCFMUSP - São Paulo - 2006

\begin{tabular}{lrr}
\hline $\begin{array}{l}\text { Localização da ponta do } \\
\text { cateter pós-tração }\end{array}$ & N & \% \\
\hline Terço inferior da veia cava superior & 9 & 69,2 \\
Terço médio da veia cava superior & 3 & 23,1 \\
Terço superior da veia cava superior & 1 & 7,7 \\
\hline Total & $\mathbf{1 3}$ & $\mathbf{1 0 0 , 0}$ \\
\hline
\end{tabular}

Dos vinte e sete cateteres inseridos, vinte e quatro foram fixados com as pontas posicionadas central ou perifericamente, como mostram os dados da Tabela 5, a seguir:

Tabela 5 - Posicionamento da ponta do cateter PICC no momento de fixação, Berçário Anexo à Maternidade do HCFMUSP - São Paulo - 2006

\begin{tabular}{lrr}
\hline $\begin{array}{l}\text { Posicionamento da ponta } \\
\text { do cateter na fixação }\end{array}$ & N & \% \\
\hline Central & 20 & 83,3 \\
Midline & 3 & 12,5 \\
Midclavicular & 1 & 4,2 \\
\hline Total & $\mathbf{2 4}$ & $\mathbf{1 0 0 , 0}$ \\
\hline
\end{tabular}

Os dados da Tabela 5 mostram que nos cateteres submetidos à manobra de tração, as pontas foram reposicionadas e fixadas na veia cava superior, em posição central.

Verifica-se que a prevalência de cateteres centrais foi de $83,3 \%$ e $16,7 \%$ foram mantidos em posição periférica.

\section{DISCUSSÃo}

Conforme os dados de caracterização da população estudada mostram, os recém-nascidos submetidos à inserção do cateter PICC são, em sua maioria, constituídos por pré-termos de baixo peso que necessitam desse dispositivo para garantir seu crescimento e desenvolvimento, uma vez que os órgãos relacionados à sucção e nutrição ainda não estão plenamente desenvolvidos.

A prematuridade é uma das principais causas de internação nas unidades neonatais, responsáveis por elevadas taxas de morbidade e mortalidade no período perinatal ${ }^{(10)}$. 
Dispor de acesso vascular central em recém-nascidos internados em unidade de cuidados intensivos neonatais, sobretudo nos recém-nascidos pré-termos que requerem administração de alimentação parenteral durante período prolongado de internação é medida fundamental para a sobrevivência e recuperação desses neonatos ${ }^{(1)}$.

Estudo de coorte prospectiva verificou a incidência e local da posição da ponta do cateter PICC de recém-nascidos internados em Unidade de Terapia Intensiva Neonatal (UTIN) de um Hospital da Arábia Saudita durante o período de 2002 a 2004. A média de idade gestacional dos recém-nascidos naquela população foi de 27,7 semanas e a média do peso de 1.040 gramas. A média da idade do neonato na data da inserção do cateter PICC foi de 12,6 dias de vida ${ }^{(3)}$. Há uma semelhança no perfil dos recém-nascidos submetidos à inserção do cateter PICC, mostrando um predomínio de recém-nascidos pré-termos e de baixo peso.

O diagnóstico clínico que prevaleceu nos recém-nascidos submetidos ao PICC foi a Síndrome do Desconforto Respiratório (SDR) ou Doença da Membrana Hialina (DMH), com $56,8 \%$ (Tabela 1). A SDR afeta sobretudo os neonatos prematuros, com peso entre 501 a 1.500 gramas. Prematuridade, sexo masculino, asfixia perinatal são fatores de risco da SDR ${ }^{(11)}$.

Estudo retrospectivo que comparou diferentes tipos de cateter percutâneo (poliuretano, silicone, polietileno e teflon) para identificar o melhor material a ser utilizado em unidade neonatal verificou que cateteres de polietileno e teflon apresentam características parecidas, porém maior incidência para trombose de veia cava em comparação com poliuretano e silicone; maior incidência de tromboflebite no grupo de recém-nascidos que utilizara cateter de silicone que cateter de poliuretano; menor resistência do cateter de silicone comparada ao de poliuretano que, por sua vez, apresenta maior freqüência de fraturas; taxa de bacteremia maior em cateteres de teflon e polietileno. Pelo estudo verificou-se que existem vantagens e desvantagens para cada tipo de cateter ${ }^{(12)}$. Os dados corroboram os do presente estudo, no qual se identificou o uso tanto de cateter de poliuretano como de silicone e preponderou o uso de cateteres de poliuretano em razão do custo ser inferior ao do cateter de silicone. No entanto, chama atenção a taxa de $20,7 \%$ de insucesso no procedimento de inserção do cateter, o que leva a questionar se o tipo de material do cateter poderia ou não estar relacionado ao sucesso na inserção do cateter PICC.

Os motivos de insucesso das punções na inserção do cateter PICC estão relacionados ao recém-nascido de forma direta e indireta. São classificados como causas diretas os fatores relacionados à anatomia e fisiologia do neonato e constituem-se como causas indiretas relacionadas à habilidade do enfermeiro que realiza o procedimento.

A confirmação da posição da ponta do cateter pode ser visualizada por injeção de contraste radiopaco, radiografia torácica e por ultra-sonografia(13).
Estudo realizado encontrou uma taxa de sucesso na implantação do cateter PICC de $84 \%$ ao avaliar prospectivamente 41 procedimentos de inserção do cateter PICC em recém-nascidos internados em UTI neonatal(3).

A ponta do cateter quando mal posicionada pode trazer sérias complicações. Uma delas é a arritmia cardíaca que ocorre, quando a ponta do cateter está abaixo do átrio direito ou abaixo do ventrículo direito ${ }^{(14)}$.

O risco para formação de trombos e flebite aumenta, quando a ponta do cateter está na entrada da veia cava superior ${ }^{(15)}$.

Do grupo de recém-nascidos cujos cateteres foram tracionados, nove $(69,2 \%)$ tiveram as pontas dos cateteres reposicionadas para o terço inferior da veia cava superior e quatro $(30,8 \%)$ no terço superior e médio da veia cava (Tabela 4).

Estudo sobre a localização da ponta do cateter PICC encontrou freqüência que variou de $25 \%$ a $40 \%$ de falhas no posicionamento da ponta do cateter PICC nas tentativas de punção venosa. As pontas posicionadas em veia axilar, subclávia e inominada apresentam $60 \%$ de chance de ocorrência de trombose, e em veia cava superior, $21 \%{ }^{(16)}$.

O comprimento do cateter PICC a ser inserido depende da veia e do membro escolhido, em média de 10 a 15 centímetros ${ }^{(17)}$.

Cabe ao enfermeiro a responsabilidade em assistir todos os pacientes com cateter PICC incluindo a checagem da posição da ponta do cateter pela radiografia de tórax ${ }^{(18)}$.

\section{CONCLUSÃO}

Em sua maioria, os recém-nascidos submetidos à inserção do cateter PICC foram do sexo masculino, com idade gestacional ao nascimento $=32$ semanas, com peso inferior a 1.500 gramas, sendo submetidos ao procedimento de inserção do cateter PICC na primeira semana de vida por indicação de infusão de Nutrição Parenteral Total. A prevalência de sucesso na implantação do cateter foi de $64,9 \%$ e a ponta do cateter foi posicionada centralmente em $54,1 \%$ dos procedimentos, abaixo dos achados encontrados na literatura. O mau posicionamento inicial da ponta do cateter esteve relacionado à introdução de comprimento do cateter, além do necessário que levou as enfermeiras a realizar manobras de extração para reposicionamento da ponta. Este dado aponta para a necessidade de uma revisão da técnica adotada para mensurar o comprimento do cateter. Outro ponto a ser mencionado foi a necessidade de realização de novos estudos, comparando as taxas de sucesso na inserção do cateter de poliuretano com o de silicone, já que a taxa de prevalência de sucesso na inserção do cateter encontrada neste estudo foi inferior aos dados da literatura, destacando que na literatura predominam estudos utilizando cateteres de silicone e neste estudo predominou uso de cateteres de poliuretano. 


\section{REFERÊNCIAS}

1. Sastre JBL, Colomer BF, Cotallo GDC, Aparício AR. Estudio prospectivo sobre catéteres epicutáneos en neonatos. An Esp Pediatr. 2000;53(2):138-47.

2. Racadio JM, Doellman DA, Johnson ND, Bean JA, Jacobs BR. Pediatric peripherally inserted central catheters: complication rates related to catheter tip location. Pediatrics. 2001;107(2):E28.

3. Tawil KA, Eldemerdash A, Hathlol KA, Laimoun BA. Peripherally inserted central venous catheters in newborn infants: malpositioning and spontaneous correction of catheter tips. Am J Perinatol. 2006;23(1):37-40.

4. Chlebicki MP, Teo EK. Review of peripherally inserted central catheters in the Singapore acute-care hospital. Singapore Med J. $2003 ; 44(10): 531-5$.

5. Camara D. Minimizing risks associated with peripherally inserted central catheter in the NICU. Am J Mater Child Nurs. 2001; 26(1):17-21.

6. Toma E. Avaliação do uso do PICC - Cateter Central de Inserção Periférica - em recém-nascidos [tese]. São Paulo: Escola de Enfermagem, Universidade de São Paulo; 2004.

7. Fricke BL, Racadio JM, Duckworth T, Donnelly LF, Tamer RM, Johnson ND. Placement of peripherally inserted central catheters without fluoroscopy in children: initial catheter tip position. Radiology. 2005;234(3):887-92.

8. Chemaly RF, Parres JB, Rehm SJ, Adal KA, Lisgaris MV, Katz-Scott $\mathrm{DS}$, et al. Venous thrombosis associated with peripherally inserted central catheters: a retrospective analysis of the Cleveland Clinic experience. Clin Infect Dis. 2002;34(9):1179-83.
9. Nadroo AM, Glass RB, Lin J, Green RS, Holzman IR. Changes in upper extremity position cause migration of peripherally inserted central catheters in neonates. Pediatrics. 2002;110(1):131-6.

10. Gaíva MAM, Gomes MMF. Cuidando do neonato: uma abordagem de enfermagem. Goiânia: AB; 2003. O prematuro; p. 35-41.

12. Evans M, Lentsch D. Percutaneously inserted polyurethane central catheters in the NICU: one unit's experience. Neonatal Netw. 1999;18(6):37-46.

13. Kaye R, Sane SS, Towbin RB. Pediatric intervention: an update - part II. J Vasc Interv Radiol. 2000;11(7):807-22.

14. Geddes $L A B$, Nichols HA. An overview of peripherally inserted central catheters. Adv Pract Nurs J. 2005;5(3):1-9

15. Vesely TM. Central venous catheter tip position: a continuing controversy. J Vasc Interven Radiol. 2003;14(5):527-34.

16. Galloway S, Bodenham A. Long-term central venous access. Br J Anaesth. 2004;92(5):722-34.

17. Pérez EEUM, Faunes M, Avaca M, Torres V, Galleguillos J, Solis L, et al. Uso de cateter central insertado periféricamente por via percutánea em recién nacido. Pediatr Día. 2002;18(1):27-31.

18. Fong $\mathrm{NI}$, Holtzman SR, Bettmann MA, Bettis SJ. Peripherally inserted central catheters: outcome as a function of the operator. J Vasc Interv Radiol. 2001;12(6):723-9. 